University of Wollongong

Research Online

Faculty of Engineering and Information

Faculty of Engineering and Information

Sciences - Papers: Part A

Sciences

$1-1-2013$

Performance evaluation of cooperative communications for STFC MBOFDM UWB

Zixuan Lin

University of Wollongong, z1715@uowmail.edu.au

Le Chung Tran

University of Wollongong, Ictran@uow.edu.au

Farzad Safaei

University of Wollongong, farzad@uow.edu.au

Follow this and additional works at: https://ro.uow.edu.au/eispapers

Part of the Engineering Commons, and the Science and Technology Studies Commons

Research Online is the open access institutional repository for the University of Wollongong. For further information contact the UOW Library: research-pubs@uow.edu.au 


\title{
Performance evaluation of cooperative communications for STFC MB-OFDM UWB
}

\begin{abstract}
Recently, the combination of cooperative communication, Space-Time-Frequency Codes (STFCs) and Multiband OFDM Ultra-Wideband (MB-OFDM UWB) has been proposed to improve the data rate, system capacity and reliability. This paper provides insightful performance evaluation for our previous proposed cooperative communication schemes for MBOFDM UWB systems. In particular, this paper shows that the usefulness of cooperative communication schemes is decided by the mutual relation between the signalto-noise ratio (SNR) of the inter-node links, referred to as inter-node SNR, and that of the uplinks, which is referred to as uplink SNR. For a certain uplink SNR value, cooperative communication becomes useful when the inter-node SNR is above a certain threshold, the so-called cooperative SNR value, and vice versa. The cooperative SNR values corresponding to different uplink conditions have been derived from the simulation results for the UWB channel models $\mathrm{CM} 1$ and $\mathrm{CM} 2$ for illustration. Two important observations drawn from this paper are that, in some cases, cooperative communication for MB-OFDM UWB might be still beneficial even when the inter-node links are relatively noisy, and/or when the source nodes are located further apart from each other than from the destination node; and this benefit over a noncooperative counterpart is more significant when the uplink channels are more dispersive.
\end{abstract}

\section{Keywords}

era2015, performance, ofdm, communications, mb, cooperative, uwb, stfc, evaluation

\section{Disciplines}

Engineering | Science and Technology Studies

\section{Publication Details}

Z. Lin, L. Chung. Tran \& F. Safaei, "Performance evaluation of cooperative communications for STFC MBOFDM UWB," in IEEE International Symposium on Communications and Information Technologies, 2013, pp. 46-51. 


\title{
Performance Evaluation of Cooperative Communications for STFC MB-OFDM UWB
}

\author{
Zixuan Lin, Le Chung Tran and Farzad Safaei \\ Faculty of Informatics \\ University of Wollongong, Australia \\ \{zl715,lctran,farzad\}@uow.edu.au
}

\begin{abstract}
Recently, the combination of cooperative communication, Space-Time-Frequency Codes (STFCs) and Multiband OFDM Ultra-Wideband (MB-OFDM UWB) has been proposed to improve the data rate, system capacity and reliability. This paper provides insightful performance evaluation for our previous proposed cooperative communication schemes for MBOFDM UWB systems. In particular, this paper shows that the usefulness of cooperative communication schemes is decided by the mutual relation between the signal-to-noise ratio (SNR) of the inter-node links, referred to as inter-node SNR, and that of the uplinks, which is referred to as uplink SNR. For a certain uplink SNR value, cooperative communication becomes useful when the inter-node SNR is above a certain threshold, the so-called cooperative SNR value, and vice versa. The cooperative SNR values corresponding to different uplink conditions have been derived from the simulation results for the UWB channel models CM1 and CM2 for illustration. Two important observations drawn from this paper are that, in some cases, cooperative communication for MB-OFDM UWB might be still beneficial even when the inter-node links are relatively noisy, and/or when the source nodes are located further apart from each other than from the destination node; and this benefit over a non-cooperative counterpart is more significant when the uplink channels are more dispersive.
\end{abstract}

\section{INTRODUCTION}

Combination of the emerging technologies, namely Multiband Orthogonal Frequency Division Multiplexing UltraWideband (MB-OFDM UWB) [1], Space-Time-Frequency Codes (STFC), and Multiple-Input Multiple-Output (MIMO), which is referred to as STFC MB-OFDM UWB, has received increasing interest from researchers. The performance of the STFC MB-OFDM UWB has been examined in some publications, such as [2],[3],[4]. For the STFCs to be implemented, the transmitter is assumed to have multiple antennas in all these works. However, the source nodes (i.e., the transmitters, such as portable devices) may only be equipped with a single antenna due to their tiny physical size. Cooperative communication technique was proposed to create a virtual MIMO system, so the concept of STFC and MIMO can still be implemented by the single antennas source nodes in a MB-OFDM UWB system to achieve large diversity.

In [5],[6],[7], we proposed several cooperative communication schemes for STFC MB-OFDM UWB. Particularly, in [5], we proposed for the first time the framework of cooperative communication for MB-OFDM UWB systems with two cooperative source nodes using the Alamouti STFC, which is the extended, three-dimension version of the original Alamouti Space-Time Block Code (STBC) in [8]. In [6], we extended the idea in [5] to propose the cooperative communication concept using a 3/4-rate Orthogonal Space-Time-Frequency Code (OSTFC) for a MB-OFDM UWB system with four cooperative source nodes in order to further take advantage of a higher diversity order. In [7], we proposed for the first time cooperative communication using a full-rate Quasi-Orthogonal STFC (QO-STFC) for the system with four cooperative source nodes to increase the date rate over the approach in [6]. All these works have illustrated the preliminary usefulness of the implementation of cooperative communication and STFCs in MB-OFDM UWB systems.

However, an in-depth performance evaluation of cooperative communication for UWB systems has not been derived in all aforementioned works. Particularly, it is assumed that the signal-to-noise ratio (SNR) is the same in all links between nodes in the network. For brevity, we refer the links between the source nodes themselves to as the inter-node links and the links between the source nodes and the destination node to as the uplinks. In fact, the SNRs of the inter-node links and those of the uplinks might not be the same. Case 1 in Fig. 1 is a typical example for the case where the SNRs of the inter-node links might be better than those of the uplinks, while Case 2 is a typical example for the reverse case. The inter-node SNR significantly affects the decoding correctness at the cooperative nodes (i.e. source nodes), which in turns significantly affects to the usefulness of the implementation of STFC cooperative communication.

When exploring the mutual relation between the inter-node SNR and the uplink SNR, one could have the following questions: (i) Would the STFC cooperative communication still be useful if the inter-node SNR is worse than the uplink SNR? (ii) If yes, what is the minimum value of the inter-node SNR for a given uplink SNR that would make cooperative communication beneficial? To the best of our knowledge, the answers for these questions in the context of STFC MB-OFDM UWB systems are still missing in the literature.

Bearing in mind that the mathematical evaluation of the bit error rate of a cooperative STFC MB-UWB system is extremely complicated, in this paper, we analyse the performance of cooperative communication using the simulation approach for a two source node MB-OFDM UWB system, using our proposed order-2 orthogonal cooperative communication scheme (2-OCCS) in [5], with various inter-node SNR 


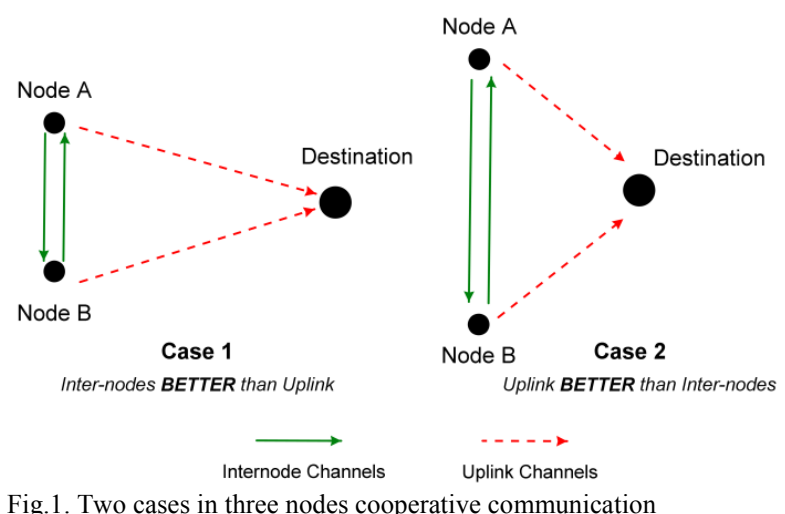

Fig.1. Two cases in three nodes cooperative communication

values and a given uplink SNR. Comparison with the error performance of a conventional MB-OFDM UWB is then derived. Simulation results show that cooperative communications is beneficial when the inter-node SNR is higher than a certain threshold, which is referred to as the cooperative SNR value hereafter. Several cooperative SNR values corresponding to different inter-node and uplink SNR conditions are also derived in the paper. The results present that even when the inter-node channels are worse than the uplink channels, cooperative communication may still be useful in the MB-OFDM UWB system in some cases.

This paper is organized as follows. Section II briefly reviews our 2-OCCS in [5]. Section III presents the system model considered in this paper. Simulation results are shown in Section IV and Section V concludes the paper.

Notations: The following notations will be used throughout the paper. The superscripts $(.)^{*}$ and $(.)^{\mathrm{T}}$ denote the complex conjugation and transposition operation, respectively. We denote $\bar{a} \bullet \bar{b}$ to be the element-wise (or Hadamard) product of the two vectors $\bar{a}$ and $\bar{b} . N_{D}$ and $N_{f f t}$ are the number of data subcarriers and the FFT/IFFT size, respectively (for MBOFDM UWB communications [1], $N_{D}=100$ and $N_{f f t}=128$ ). We refer the time required to transmit a MB-OFDM symbol to as a MB-OFDM symbol time slot (Ts), which is $312.5 \mathrm{~ns}$, including the FFT/IFFT period of $242.42 \mathrm{~ns}$ and the zero padded suffix duration of $70.08 \mathrm{~ns}$ [1].

\section{ORDER-2 ORTHOGONAL COOPERATIVE} COMMUNICATION SCHEME USING ALAMOUTI STFC (2-OCCS)

This section briefly reviews the cooperative STFC UWB scheme that we proposed in [5]. The proposed scheme is depicted in Fig.2. We consider the application of the following Alamouti STFC [2],[8], which is the extended, threedimensional version of the original Alamouti code

$$
S=\left[\begin{array}{cc}
\bar{S}_{A_{i}} & \bar{S}_{B_{i}} \\
-\bar{S}^{*} & -{ }^{*} \\
-S_{B_{i}} & S_{A_{i}}
\end{array}\right]
$$

The STFC symbols $\bar{s}_{A_{i}}$ and $\bar{s}_{B_{i}}$ are the column vectors that consist of the original modulated data (i.e., before the IFFT operation) and correspond to the $i$-th MB-OFDM symbol

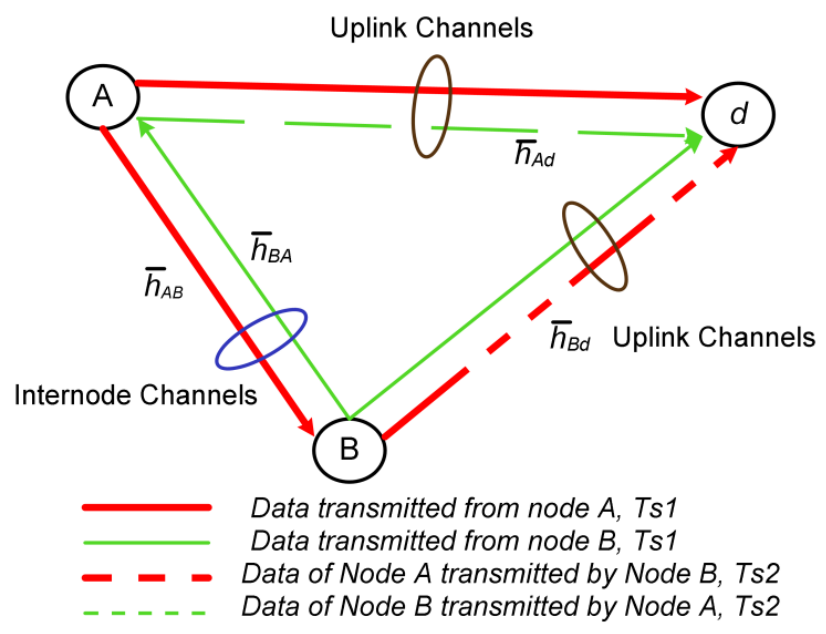

Fig.2. Cooperative communication using the Alamouti STFC in MB-OFDM UWB between the source nodes $A, B$ and the destination $d$

transmitted from Nodes $A$ and $B$, respectively. It is assumedthat nodes in the system are perfectly synchronized. Denote $\bar{h}_{j k}=\left[h_{j k, 1}, h_{j k, 2}, \ldots h_{j k, L_{j k}}\right]^{T}$ to be the channel vector between the two nodes $j$ and $k$, where $j \in\{A, B\}, k \in\{A, B, d\}$ (see Fig.2), and $L_{j k}$ is the number of multipaths in this channel. The channels between nodes are modeled as independent, log-normally distributed random variables (RVs) [9], and $\bar{h}_{j k}$ is assumed to be constant during one STFC block, but random from blocks to blocks. The channel coefficients are assumed to be known at the destination node. Each of the source nodes $A$ and $B$ and the destination node $d$ are equipped with only one antenna for transmitting and receiving signals. In the cooperative communication, each source node transmits its own data as well as performing the role of a cooperative agent for other nodes.

In the 2-OCCS, two nodes are paired to cooperate with one another. At the first MB-OFDM symbol time slot, Node $A$ broadcasts its symbol $\bar{s}_{A_{i}}$ to the destination node $d$ as well as its partner (Node $B$ ). Simultaneously, Node $B$ broadcasts its symbol $\bar{S}_{B_{i}}$ to its partner node $A$ and the destination node $d$. (To make this full duplex communication possible, a subband allocation technique was proposed in [5], which will be detailed later in this section). We denote the decoded symbols at Nodes $A$ and $B$ to be $\bar{s}_{B_{i}}$ and $\bar{s}_{A_{i}}$. In the second MB-OFDM time slot, these two source nodes retransmit the decoded symbols to the destination in the form of $-\bar{s}_{B_{i}}^{*}$ and $\bar{s}_{A_{i}}^{*}$, respectively.

After the overlap-and-add operation (OAAO) [2], [8] and FFT have been performed, the signals received at the destination node $d$ during the two time slots can be represented as

$$
\begin{aligned}
& \overline{\mathfrak{r}}_{1}=\overline{\mathfrak{h}}_{A d} \bullet \bar{S}_{A_{i}}+\overline{\mathfrak{h}}_{B d} \bullet \bar{S}_{B_{i}}+\overline{\mathfrak{n}}_{1} \\
& \overline{\mathfrak{r}}_{2}=-\overline{\mathfrak{h}}_{A d} \bullet \bar{S}_{B_{i}}^{*}+\overline{\mathfrak{h}}_{B d} \bullet \bar{S}_{A_{i}}^{*}+\overline{\mathfrak{n}}_{2}
\end{aligned}
$$

where $\overline{\mathfrak{h}}_{j k}=F F T\left(\bar{h}_{j k}\right), \overline{\mathfrak{n}}_{t}=F F T\left(\bar{n}_{t}\right)$, while $\bar{n}_{t}(t=1,2)$ denotes the column vector of complex Gaussian noise affecting the destination node during the $t$-th MB-OFDM symbol time slot. 


\begin{tabular}{|c|c|c|c|c|c|}
\hline \multirow{2}{*}{$\begin{array}{l}\text { Freq. MHz } \\
\quad 4224\end{array}$} & \\
\hline & $\overline{\mathrm{S}}_{\mathrm{B} 1}$ & $\vec{S}_{\mathrm{A} 1}{ }^{*}$ & $-\overline{\mathrm{S}}_{\mathrm{B} 2}$ & $-\boldsymbol{S}_{\mathrm{A} 2}{ }^{*}$ & Band \#2 \\
\hline 3696 & $\overline{\mathbf{S}_{\mathrm{A} 1}}$ & $-\vec{S}_{\mathrm{B} 1}{ }^{*}$ & $-\overline{\mathrm{S}}_{\mathrm{A} 2}{ }^{*}$ & $-\overrightarrow{\boldsymbol{S}}_{\mathrm{B} 2}{ }^{*}$ & Band \#1 \\
\hline \multirow{2}{*}{3168} & & \multirow{2}{*}{\multicolumn{2}{|c|}{ Node A }} & & $\overrightarrow{\text { Time }}$ \\
\hline & & & & Node B & \\
\hline
\end{tabular}

Fig.3. Subband allocation in the 2-OCCS

Denote $\overline{\mathfrak{h}}_{j k}=\left[\hbar_{j k, 1}, \hbar_{j k, 2,}, \ldots ., \hbar_{j k, N_{f j}}\right]^{T}$ and $\overline{\mathfrak{r}}_{t}=\left[\mathfrak{r}_{t, 1}, \mathfrak{r}_{t, 2}, \ldots, \mathfrak{r}_{t, N_{f f}}\right]^{T}$. Once the destination node receives the symbols transmitted during the two time slots, it is able to decode the symbols.

In the proposed system, the symbols can be decoded by the maximum likelihood (ML) decoding as detailed in [2]. Each of the two MB-OFDM symbols $\bar{s}_{A_{i}}$ and $\bar{s}_{B_{i}}$ can be decoded separately, rather than jointly, as mentioned in Table I. Furthermore, each individual modulated symbol (among $N_{\mathrm{D}}$ symbols) within the symbol $\bar{s}_{A_{i}}$ (or $\bar{S}_{B_{i}}$ ) can be decoded separately, rather than the whole $N_{\mathrm{D}}$ data being decoded simultaneously. Thus the decoding process is completely linear, and relatively simple. In particular, the decoding metrics for data at the $n$-th subcarrier, for $n=1, \ldots, N_{D}$, in the MB-OFDM symbols $\bar{s}_{A_{i}}$ and $\bar{s}_{B_{i}}$ are

$$
\begin{aligned}
S_{A_{i}, n} & =\arg \min _{s \in C}\left\{\left|\left(\hbar_{A d, n}^{*} \mathfrak{r}_{1, n}+\hbar_{B d, n} \mathfrak{r}^{*}{ }_{2, n}\right)-s\right|^{2}\right. \\
& \left.+\left[-1+\left(\left|\hbar_{A d, n}\right|^{2}+\left|\hbar_{B d, n}\right|^{2}\right)\right]|s|^{2}\right\} \\
S_{B_{i}, n} & =\arg \min _{s \in C}\left\{\left|\left(\hbar^{*}{ }_{B d, n} \mathfrak{r}_{1, n}-\hbar_{A d, n} \mathfrak{r}^{*}{ }_{2, n}\right)-s\right|^{2}\right. \\
& \left.+\left[-1+\left(\left|\hbar_{A d, n}\right|^{2}+\left|\hbar_{B d, n}\right|^{2}\right)\right]|s|^{2}\right\}
\end{aligned}
$$

The proposed 2-OCCS requires the full duplex communication between source nodes, i.e. nodes are able to transmit their own data and receive the partner's data at the same time via only one antenna. To make this possible, we took advantage of important technical specifications of MB-OFDM UWB devices that, support for the first band group $(3168-4752 \mathrm{MHz}$, see [1], Table 7-1) is mandatory, and that the Time Frequency Code (TFCs) numbers 5, 6 and 7 for the first band group are non-overlapped with each other (See [1] Table 7-2) and proposed a subband allocation technique as depicted in Fig.3. This subband allocation technique allows Node $A$ transmits the signals using TFC 5 (i.e. the radio frequency (RF) is in the range 3168 - $3696 \mathrm{MHz}$ corresponding to the subband 1 [1, Table 7-1]). Similarly, Node $B$ may transmit signals using TFC 6 (subband 2). The destination node must be able to receive the signals in all subbands 1 and 2 .

It has been shown in [5] that the 2-OCCS provides significantly better error performance, compared to the noncooperative STFC MB-OFDM system, in the case that the SNR values at inter-node links and at uplinks are all equal. This observation might be changed in a more practical scenario where, for a certain uplink SNR value, the inter-node SNR might be varied. The inter-node SNR significantly affects the preciseness of the decoding processes at the source nodes, thus
TABLE I

2-OCCS DECODING METRICS PSK OR QAM MODULATION

\begin{tabular}{c|c}
\hline Symbol & Decoding Metric \\
\hline $\bar{S}_{A}$ & $\arg \min _{s \in C N_{D}} \|\left\{\left|\left(\overline{\mathfrak{h}}_{A d}^{*} \bullet \overline{\mathfrak{r}}_{1}+\overline{\mathfrak{h}}_{B d} \bullet \overline{\mathfrak{r}}_{2}^{*}\right)-\bar{s}\right| \cdot \cdot^{\wedge} 2\right.$ \\
& $\left.+\left[-\overline{1}+\left(\left|\overline{\mathfrak{h}}_{A d}\right| \cdot \cdot^{\wedge} 2+\left|\overline{\mathfrak{h}}_{B d}\right| \cdot \wedge 2\right)\right] \bullet\left(|\bar{s}| \cdot \cdot^{\wedge} 2\right)\right\}||_{F}^{2}$ \\
\hline $\bar{s}_{B}$ & $\arg \min _{s \in C N_{D}} \|\left\{\left|\left(\overline{\mathfrak{h}}_{B d}^{*} \bullet \overline{\mathfrak{r}}_{1}-\overline{\mathfrak{h}}_{A d} \bullet \overline{\mathfrak{r}}_{2}^{*}\right)-\bar{s}\right| \cdot^{\wedge} 2\right.$ \\
& $\left.+\left[-\overline{1}+\left(\left|\overline{\mathfrak{h}}_{A d}\right| \cdot \cdot^{\wedge} 2+\left|\overline{\mathfrak{h}}_{B d}\right| \cdot \wedge 2\right)\right] \bullet(|\bar{s}| \cdot \wedge 2)\right\} \|_{F}^{2}$ \\
\hline
\end{tabular}

having significant impact on the overall system error performance. As the result, the mutual relationship between the uplink SNR and the inter-node SNR decides whether the implementation of cooperative communication is beneficial.

In the rest of the paper, we present in detail the system model used to examine the effect of the inter-node links to the overall system error performance for a given uplink SNR. We shall demonstrate by the simulation results that, for a certain uplink SNR, cooperative communication becomes useful when the inter-node SNR starts to be larger than a threshold value, referred to as the cooperative SNR value. Several threshold values are then derived from the simulation results, depending on different uplink SNR conditions. These threshold values are the lower bound of the inter-node SNRs in order for the implementation of cooperative communication in MBOFDM UWB to be useful. It is noted that, while the derivation of the cooperative SNR values from simulation results is relatively simple, the derivation of their mathematical values is extremely complicated, since the channels are log-normally distributed, and thus being out of the scope of this paper.

\section{2-OCCS SYSTEM MODEL WITH VARIED UPLINK AND INTER-NODE CONDITIONS}

In this section, we will present the system model applied in this paper. The system model is similar to Fig.2, except that SNRs in the inter-node links are not equal to those in the uplinks. Instead, uplinks have a certain SNR value, denoted as $\mathrm{SNR}_{\text {up }}$. For the illustration purpose, we assume $\mathrm{SNR}_{\text {up }}$ be in the range from $5 \mathrm{~dB}$ to $25 \mathrm{~dB}$. For each $\mathrm{SNR}_{\text {up }}$, the inter-node $\mathrm{SNR}$, denoted as $\mathrm{SNR}_{\text {int }}$, is varied between $0 \mathrm{~dB}$ and $30 \mathrm{~dB}$.

As explained in Section II, two source nodes broadcast their own MB-OFDM symbols to the destination (and each other) in the first time slot. Thus, these two source nodes are acting like a conventional SISO (Single-Input Single-Output) MBOFDM UWB system in the first time slot. The inter-node channel vectors $\bar{h}_{A B}$ and $\bar{h}_{B A}$ between the source nodes are modeled as independently log-normally distributed RVs. Denote $\bar{h}_{m n}=\left[h_{m n, 1}, h_{m n, 2}, \ldots h_{m n, L_{m n}}\right]^{T}$ to be the channel vector between two nodes $n$ and $m$, where $m \in\{A, B\}, n \in\{A, B\}$ and $L_{m n}$ is the number of multipaths in this link. Two source nodes are assumed to be relatively close to each other, be- 
tween 0 - 4 meters with a line of sight (LOS). Therefore, the channel model CM1 could be applied to the inter-node links [9]. Unlike the inter-node links, the uplinks might or might not have LOS, thus either CM1 or CM2 will be used to model the uplink channels.

As shown in Fig.3, the 2-OCCS requires two MB-OFDM symbol time slots to transmit the Alamouti STFC in two different subbands. In the first MB-OFDM time slot, Node $A(B)$ broadcasts its symbol $\bar{s}_{A_{i}}\left(\bar{S}_{B_{i}}\right)$ in the subband 1 (subband 2). In the second MB-OFDM time slot, the source nodes A and B retransmit the decoded symbols to the destination in the form of $-\bar{s}_{B_{i}}^{*}$ and $\bar{s}_{A_{i}}^{*}$, thus effectively constructing an Alamouti STFC matrix. The decoding procedures in the destination node $d$ are presented in Equations (2) and (3).

As the SNR value in the inter-node channels, $\mathrm{SNR}_{\text {int }}$, is varied, the decoding accuracy at the source node also changes. The decoded vectors $\bar{s}_{A_{i}}$ and $\bar{s}_{B_{i}}$ might not be exactly the same as $\bar{S}_{A_{i}}$ and $\bar{S}_{B_{i}}$ if $\mathrm{SNR}_{\text {int }}$ is relatively low. The errors occurred in the decoded vectors $\bar{S}_{A_{i}}$ and $\bar{S}_{B_{i}}$ will definitely affect the bit error performance at the destination node as the system require all the symbols transmitted during two time slots to decode the original symbols $\bar{S}_{A_{i}}$ and $\bar{S}_{B_{i}}$.

In Section IV, we will investigate the performance of cooperative communication in different inter-node and uplink channel conditions in order to determine a) how $\mathrm{SNR}_{\text {int }}$ affects the overall system performance and $b$ ) at what $\mathrm{SNR}_{\text {int }}$ values the implementation of cooperative communication in the MBOFDM UWB system is useful.

\section{Simulation Results}

To evaluate the performance advantage of cooperative communication, we ran several Monte-Carlo simulations for the 2-OCCS and conventional MB-OFDM UWB systems. Each run of simulations was carried out with 1200 MB-OFDM symbols. One hundred channel realizations of each channel model (CM1 and CM2) were considered for the transmission of each MB-OFDM symbol. In simulations, SNR (either $\mathrm{SNR}_{\text {int }}$ or $\mathrm{SNR}_{\text {up }}$ ) is defined to be the signal-to-noise ratio (dB) per sample in a MB-OFDM symbol at each Rx antenna.

In order to fairly compare the error performances of the non-cooperative and 2-OCCS systems, the following constraints are applied to all simulations.

Power constraint: The total received power at each $\mathrm{Rx}$ antenna at the destination during each time slot need to be the same in both systems. Therefore, the signal constellation points in the 2-OCCS are scaled down by a factor of $1 / \sqrt{2}$.

Data rate constraint: The same signal constellation mapping (QPSK) scheme is applied to the two systems to guarantee the same bit rate.

We assume the two uplinks have the same SNR, denoted as $\mathrm{SNR}_{\text {up. }}$. For each $\mathrm{SNR}_{\text {up }}$, we vary the SNR value in the internode links, denoted as $\mathrm{SNR}_{\text {int }}$, and record the bit error rate performance for the 2-OCCS. Meanwhile, we also simulate the conventional SISO MB-OFDM UWB system with the
TABLE II

COOPERATIVE SNRS In CHANNEL MODEL CM1 \& CM2

\begin{tabular}{|c|c|c|}
\hline $\begin{array}{c}\text { Uplink SNR } \\
\left(\mathrm{SNR}_{\mathrm{up}}\right)\end{array}$ & $\begin{array}{c}\text { CM1 Cooperative } \\
\text { SNR }\end{array}$ & $\begin{array}{c}\text { CM2 Cooperative } \\
\text { SNR }\end{array}$ \\
\hline $5 \mathrm{~dB}$ & $6.3 \mathrm{~dB}$ & $5.6 \mathrm{~dB}$ \\
\hline $10 \mathrm{~dB}$ & $11.3 \mathrm{~dB}$ & $10.1 \mathrm{~dB}$ \\
\hline $15 \mathrm{~dB}$ & $14.4 \mathrm{~dB}$ & $12.7 \mathrm{~dB}$ \\
\hline $20 \mathrm{~dB}$ & $15.8 \mathrm{~dB}$ & $14.6 \mathrm{~dB}$ \\
\hline $25 \mathrm{~dB}$ & $17.0 \mathrm{~dB}$ & $15.1 \mathrm{~dB}$ \\
\hline
\end{tabular}

same uplink channel condition, i.e., having the SNR equal to $\mathrm{SNR}_{\text {up }}$ in the 2-OCCS

. The two performances are then compared, and the lower bound of the cooperative SNR values can be estimated.

Fig.4 (5) compares the error performances of the 2-OCCS and the conventional MB-OFDM with $\mathrm{SNR}_{\text {up }}$ being 5, 10, 15, 20 and $25 \mathrm{~dB}$ and $\mathrm{SNR}_{\text {int }}$ ranging from 0 to $30 \mathrm{~dB}$ in the channel model CM1 (CM2). The performance of the conventional MB-OFDM system (i.e., SISO MB-OFDM system) is presented as dotted lines which are in parallel with the horizontal axis. This is because the performance of the conventional MBOFDM system does not depend on $\mathrm{SNR}_{\text {int }}$, but only on $\mathrm{SNR}_{\text {up }}$. Therefore, the system bit error rate (BER) is constant for a given value of $\mathrm{SNR}_{\mathrm{up}}$.

From these figures, it is clear that the 2-OCCS provides a better error performance than the conventional system when $\mathrm{SNR}_{\text {int }}$ is above a certain value, referred to as the cooperative SNR. The cooperative SNR value can be determined by the intersection between the two error performance curves of the SISO MB-OFDM system and the 2-OCCS one corresponding to the same $\mathrm{SNR}_{\text {up. }}$. The cooperative $\mathrm{SNR}$ values are summarised in Table II. Recall that the cooperative SNR values are the lower bound of the inter-node SNR values, corresponding to given uplink SNR values, in order for the implementation of cooperative communication to be useful, compared to the noncooperative (i.e., conventional) MB-OFDM UWB system.

It can also be seen from Table II that, for a given $\mathrm{SNR}_{\text {up }}$, the cooperative SNRs in CM2 are always smaller than those in CM1. Note that CM2 is much more dispersive than CM1 [9]. Additionally, Figures 4 and 5 show that the performance of cooperative communication in CM1 is always better than that

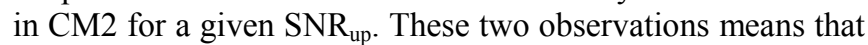
the benefit of the implementation of cooperative communication in MB-OFDM UWB systems over the conventional system occurs earlier and is more significant when the uplinks are more dispersive.

To have more insights of the cooperative SNR values, we represent the cooperative SNR values for CM1 and CM2 mentioned in Table II in more detail in Fig.6. Clearly, the cooperative SNR values decrease significantly in the more dispersive channel model. This means that the threshold SNR value where the implementation of cooperative communication becomes useful is reached earlier in the case of more dispersive uplink channels.

In Fig.6, the dotted line is added to this figure to indicate the boundary where $\mathrm{SNR}_{\text {up }}=\mathrm{SNR}_{\text {int }}$. Two reference points, $\mathrm{A}$ and 


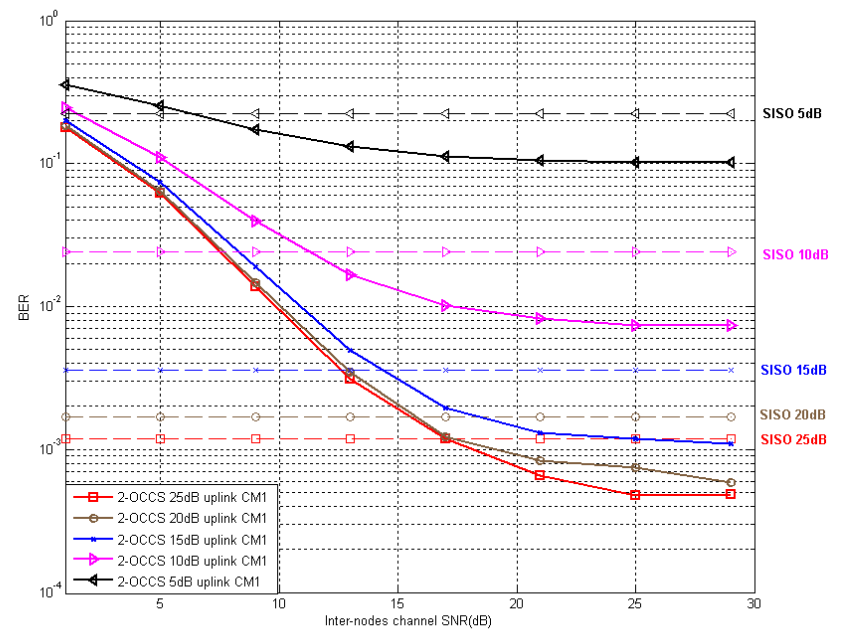

Fig.4. Cooperative communication vs. SISO MB-OFDM UWB in CM1

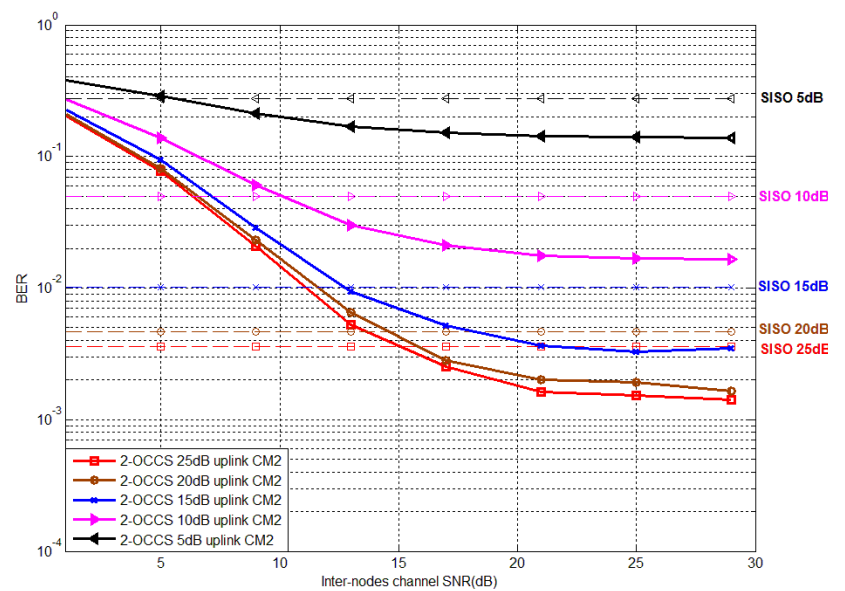

Fig.5. Cooperative communication vs. SISO UWB in CM2

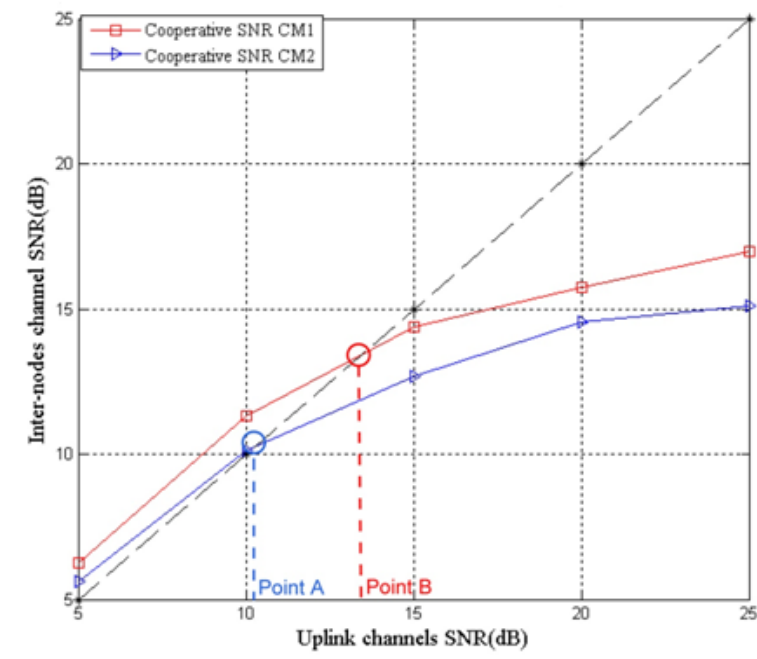

Fig.6. $\mathrm{SNR}_{\text {int }}$ vs. $\mathrm{SNR}_{\text {up }}$ in $\mathrm{CM} 1$ and $\mathrm{CM} 2$
$\mathrm{B}$, are the intersections between the dotted line and the cooperative SNR curves for CM2 and CM1, respectively. Denote the $\mathrm{SNR}_{\text {up }}$ at these reference points to be $\mathrm{SNR}^{\mathrm{A}}$ up and $\mathrm{SNR}^{\mathrm{B}}$ up.

It can be observed that, when the uplink SNR is lower than the $\mathrm{SNR}^{\mathrm{A}}$ up (similarly for $\mathrm{SNR}^{\mathrm{B}}$ up in $\mathrm{CM} 1$ ) (i.e., on the left side of the reference point), $\mathrm{SNR}_{\text {int }}$ should be slightly higher than $\mathrm{SNR}_{\text {up }}$ (the two curves are above the dotted line) in order for cooperative communication to have better performance than the conventional SISO MB-OFDM system. For instance, given $\mathrm{SNR}_{\text {up }}=8.5 \mathrm{~dB}$, cooperative communication is only useful if the inter-node SNR is not smaller than $8.6 \mathrm{~dB}(10 \mathrm{~dB})$ in $\mathrm{CM} 2$ (CM1). Case 1 in Fig. 1 is a typical example for the scenario that $\mathrm{SNR}_{\text {int }}$ is higher than $\mathrm{SNR}_{\text {up }}$, where two source nodes are relatively closer to their partner than to the destination.

However, when the uplink SNR value is higher than the SNR at the reference points (right hand side of the reference points), cooperative communication is always useful despite $\mathrm{SNR}_{\text {int }}$ being smaller than $\mathrm{SNR}_{\text {up }}$ (the two curves are below the dotted line). $\mathrm{SNR}_{\text {int }}$ is smaller than $\mathrm{SNR}_{\text {up }}$ in the following two typical scenarios. First, the two source nodes might actually be located further from each other than from the destination (cf. Case 2 in Fig.1). Second, the inter-node links might be noisier than the uplinks, even though the source nodes might be closer to each other, compared to the destination. . In other words, when the uplink channel condition is better, cooperation between the noisy source nodes can still provide better performance than the non-cooperative system.

\section{CONCLUSIONS}

This paper provides insightful performance analysis for our previously proposed cooperative communication scheme (2OCCS) for MB-OFDM UWB systems in a more practical scenario where the usefulness of cooperative communication is decided by the mutual relation between the inter-node SNR and the uplink SNR. It is concluded that the implementation of cooperative communication starts to be useful when the internode SNR reaches a certain threshold, referred to as the cooperative SNR value. Several cooperative SNR values for different uplink SNR conditions have been derived from the simulation results for CM1 and CM2 for illustration. It is also shown that cooperative communication for MB-OFDM UWB is useful even when the inter-node links are noisy, or when the source nodes are located further apart from each other than from the destination node. The benefit of the implementation of cooperative communication is more significant for the more dispersive uplinks. Extension of this work to derive the exact mathematical formula for the cooperative SNR values would be our future work.

\section{REFERENCES}

[1] WiMedia, "Multiband OFDM physical layer specification," WiMedia Alliance, Release 1.1, July 2005.

[2] L.C.Tran and A. Mertins, "Space-Time-Frequency Code implementation in MB-OFDM UWB communications: Design criteria and performance", 
IEEE Transactions on Wireless Communications, vol. 8, no. 2, pp.701713, Feb.2009.

[3] L. C. Tran, A. Mertins, E. Dutkiewicz, and X. Huang, "Space-timefrequency codes in MB-OFDM UWB communications: Advanced order-8 STFC and its performance," Proc. 7th IEEE International Symposium on Communications and Information Technologies (ISCIT), Oct.2007.

[4] T.-H. Tan and K.-C. Lin, "Performance of space-time block coded MBOFDM UWB systems," Proc. 4th Annual Communication Networks and Services Research Conference (CNSR), pp. 323 - 327, May.2006.

[5] L. C. Tran, A. Mertins, and T. A. Wysocki, "Cooperative Communication in Space-Time-Frequency Coded MB-OFDM UWB",Proc. 68th IEEE Vehicular Technology Conference (VTC), pp. 15, Sept.2008.

[6] Z. Lin, L. C. Tran, and F. Safaei, "Order-4 Orthogonal Cooperative Communication in Space-Time-Frequency Coded MB-OFDM UWB," Proc. 12nd IEEE International Symposium on Communications and Information Technologies (ISCIT), pp. 1-5, Oct.2012.

[7] Z. Lin, L. C. Tran, F. Safaei and T. A. Wysocki, "Order-4 QuasiOrthogonal Cooperative Communication in STBC MB-OFDM UWB," Proc. 6th International Conference on Signal Processing and Communication Systems (ICSPCS), pp.1-6. Dec.2012.

[8] S. M. Alamouti, "A simple transmit diversity technique for wireless communications", IEEE Journal on Selected Areas in Communications, vol. 16, no. 8, pp. 1451-1458, Oct.1998.

[9] J. Foerster et. al., "Channel modelling sub-committee report final," IEEE P802.15 Working Group for Wireless Personal Area Networks (WPANs), IEEE P802.15-02/490r1-SG3a, Oct.2005. 